\title{
Bioreactor virome metagenomics sequencing using DNA spike-ins
}

\author{
Geert Cremers $^{1}{ }^{\text {, }}$ Lavinia Gambelli ${ }^{1}$, Theo van Alen ${ }^{1}$, Laura van Niftrik ${ }^{1}$, Huub JM Op den Camp ${ }^{\text {Corresp. }}{ }^{1}$ \\ ${ }^{1}$ Department of Microbiology, Institute of Water and Wetland Research, Faculty of Science, Radboud University, Nijmegen, Netherlands \\ Corresponding Author: Huub JM Op den Camp \\ Email address: h.opdencamp@science.ru.nl
}

With the emergence of Next Generation Sequencing, major advances were made with regard to identifying viruses in natural environments. However, bioinformatical research on viruses is still limited because of the low amounts of viral DNA that can be obtained for analysis. To overcome this limitation, DNA is often amplified with multiple displacement amplification (MDA), which may cause an unavoidable bias. Here, we describe a case study in which the virome of a bioreactor is sequenced using lon Torrent technology . DNAspiking of samples is compared with MDA-amplified samples. DNA for spiking was obtained by amplifying a bacterial 16S rRNA gene. After sequencing, the 16S rRNA gene reads were removed by mapping to the Silva database. Three samples were tested, a whole genome from Enterobacteria P1 Phage and 2 viral metagenomes from an infected bioreactor. For one sample, the new DNA-spiking protocol was compared with the MDA technique. When MDA was applied, the overall GC content of the reads showed a bias towards lower GC\%, indicating a change in composition of the DNA sample. Assemblies using all available reads from both MDA and the DNA-spiked samples resulted in six viral genomes. All six genomes could be almost completely retrieved (97.9\%-100\%) when mapping the reads from the DNA-spiked sample to those six genomes . In contrast, $6.3 \%-77.7 \%$ of three viral genomes was covered by reads obtained using the MDA amplification method and only three were nearly fully covered (97.4\%-100\%). This case study shows that DNA-spiking could be a simple and inexpensive alternative with very low bias for sequencing of metagenomes for which low amounts of DNA are available. 
1 Bioreactor virome metagenomics sequencing using DNA 2 spike-ins

3

4 Geert Cremers, Lavinia Gambelli, Theo van Alen, Laura van Niftrik \& Huub J.M.

5 Op den Camp*

6 Department of Microbiology, Institute for Water and Wetland Research, Faculty of Science,

7 Radboud University, Nijmegen, the Netherlands.

8 *Corresponding author:

9 h.opdencamp@science.ru.nl

10 Heyendaalseweg 135, NL 6525 AJ Nijmegen, The Netherlands 
11

12

13

14

15

16

17

18

19

20

21

22

23

24

25

26

27

\section{Abstract}

With the emergence of Next Generation Sequencing, major advances were made with regard to identifying viruses in natural environments. However, bioinformatical research on viruses is still limited because of the low amounts of viral DNA that can be obtained for analysis. To overcome this limitation, DNA is often amplified with multiple displacement amplification (MDA), which may cause an unavoidable bias. Here, we describe a case study in which the virome of a bioreactor is sequenced using Ion Torrent technology. DNA-spiking of samples is compared with MDA-amplified samples. DNA for spiking was obtained by amplifying a bacterial 16S rRNA gene. After sequencing, the $16 \mathrm{~S}$ rRNA gene reads were removed by mapping to the Silva database. Three samples were tested, a whole genome from Enterobacteria P1 Phage and 2 viral metagenomes from an infected bioreactor. For one sample, the new DNA-spiking protocol was compared with the MDA technique. When MDA was applied, the overall GC content of the reads showed a bias towards lower $\mathrm{GC} \%$, indicating a change in composition of the DNA sample. Assemblies using all available reads from both MDA and the DNA-spiked samples resulted in six viral genomes. All six genomes could be almost completely retrieved (97.9\%$100 \%$ ) when mapping the reads from the DNA-spiked sample to those six genomes. In contrast, $6.3 \%-77.7 \%$ of three viral genomes was covered by reads obtained using the MDA amplification method and only three were nearly fully covered $(97.4 \%-100 \%)$. This case study shows that DNA-spiking could be a simple and inexpensive alternative with very low bias for sequencing of metagenomes for which low amounts of DNA are available.

\section{Introduction}

Microbial research has been mainly culture-based since the work of Pasteur and Koch. This has led to great improvement of our knowledge of the microbial and viral world. However, our knowledge is probably still only the tip of the iceberg, as most of the microorganisms cannot be cultured (Rosario \& Breitbart, 2011). In recent years, there has been a greater focus on the hidden bacterial and viral 'black matter' (Krishnamurthy \& Wang 2017) since the development of next generation sequencing (NGS) techniques which allow the determination of the microbial community without the need for cultivation. Without the necessity of cultivation prior to sequencing, organisms that cannot be cultured under artificial conditions are now being sequenced in increasing numbers. This is especially true for bacteria; sequencing viral black matter from environmental samples is still hampered by a variety of factors. Besides the obvious problem that not all viruses are DNA viruses (Steward et al., 2013), there is also the challenge of the low quantity of DNA that can be retrieved from viruses. Although viruses outnumber bacteria 5 to 25 times in numbers (Fuhrman, 1999; Clokie et al., 2011), the fact that viruses have on average a significantly smaller genome size means that the viral DNA yield from any given sample is significantly lower compared to bacterial DNA (approximately tens of attograms per virion vs femtograms per bacterium) (Brum \& Sullivan, 2015). Because of this, DNA is often extracted using DNA extraction methods optimized for virions gathered from a large amount 
(20-200 L) of sample (Breitbart et al., 2002; Thurber et al., 2009; Duhaime, 2012; Steward et al., 2013).

Since sampling large amounts is not always possible and because there is loss of DNA in every step of DNA isolation protocols, the final yield of viral DNA remains low. Therefore, two options are available for sequencing. A) Sequence very low quantities (femtograms to picograms of DNA). However, this method is sensitive to contamination and might skew community composition. It also complicates binning and assembly afterwards because of the lack of template DNA (Rinke et al., 2016). B) Amplify the low amount of DNA before sequencing. Several methods are available for this purpose (Duhaime et al., 2012; Brum \& Sullivan, 2015), with the Multiple Displacement Amplification (MDA) method being the most widely used. However, MDA has a few drawbacks. Because it is amplification, it unavoidably introduces a bias (Kim \& Bae, 2011; Marine et al., 2014) and furthermore information of relative abundance in the original sample is lost (Yilmaz et al., 2010). In practical terms, there is a high potential of cross contamination throughout the lab, which is particularly problematic in a laboratory where viral work is the main area of research.

In view of the growing interest in the impact of viruses on ecosystems, there is a growing need for bias-limited and preferably easy sequencing. However, the recurring problem on most sequencing platforms is the relative large amount ( $1 \mathrm{ng}$ to $1 \mu \mathrm{g}$ depending on the sequencing platform) of DNA needed for the library preparation for sequencing compared to the often low yield of DNA from viral population samples (less than $1 \mathrm{ng}$ ). Besides the two options discussed above, a theoretical third option would be to artificially raise the DNA concentration by adding DNA that is naturally non-occurring in any virus. Prime candidate would be the bacterial $16 \mathrm{~S}$ ribosomal gene as it has not been found in any known virus up to date. In this report we describe a case study for metagenome sequencing with the Ion Torrent Personal Genome Machine in which we spiked $16 \mathrm{~S}$ ribosomal DNA to low amounts $(0.1,5$ and $7 \mathrm{ng})$ of DNA from different samples. One of those samples was a bacteriophage population that we compared with traditional MDA amplification.

\section{Material and methods}

To test the technical feasibility of our approach, the protocol was performed three times: once with Enterobacteria Phage P1 (P1-spiked), once with a viral metagenome extracted from an infected bioreactor with a general extraction protocol (DNA-spiked-GP) and finally with a viral metagenome from the same infected bioreactor extracted with a specialised protocol for viral metagenomic DNA (DNA-spiked-SP). The last dataset was also sequenced after MDA amplification.

\section{Sample collection and DNA extraction: general protocol}

The bacteriophage population used for sequencing was obtained from a Methylomirabilis oxyfera bioreactor enrichment culture. A sample of $4 \mathrm{~L}$ of bioreactor effluent was collected over a period 
of time of 20 days and was stored at $4{ }^{\circ} \mathrm{C}$. To concentrate the viral particles, the sample was first filtered through a $0.22 \mu \mathrm{m}$ filter (nucleopore track-etched polycarbonate membrane filters, Whatman) and then further concentrated using $30 \mathrm{kDa}$ Vivaspin Spin Columns (GE Healthcare Life Sciences), to a final volume of $3 \mathrm{ml}$. A P1 reference bacteriophage (genome sequence NC_005856.1) was used as a positive control for DNA extraction. The DNA extraction was performed according to the protocol published by Thurber et al. (2009). Using the Qubit dsDNA HS assay kit (Life), the extracted DNA was quantified at $146 \mathrm{ng} / \mathrm{ml}$.

\section{Sample collection and DNA extraction: specialised protocol}

The bacteriophage population used for sequencing was obtained from the same M. oxyfera bioreactor enrichment culture. See Gambelli et al. (2016) for a full description. Bioreactor material was collected over a period of about three months, stored at $4{ }^{\circ} \mathrm{C}$ and viral particles were obtained as described before (Gambelli et al., 2016). Briefly, the aggregated microbial biomass was disrupted to free the bacteriophages and viral particles were precipitated using PEG8000 (Guo et al., 2012). Free bacteriophages present in the bioreactor supernatant medium and not within the bacterial aggregates were precipitated by iron chloride flocculation (Cunningham et al., 2015).

The two samples obtained by iron chloride flocculation and PEG 8000 precipitation were pooled together and bacteriophages were concentrated by ultracentrifugation (Optima XE90, BeckmanCoulter; Rotor: Type $90 \mathrm{Ti}$, Beckman-Coulter) at $77,000 \mathrm{x} \mathrm{g}$ at $4^{\circ} \mathrm{C}$ for $1 \mathrm{~h}$. The pellet was resuspended in $1 \mathrm{ml}$ of supernatant and the total DNA was extracted according to the protocol published by Thurber et al. (2009). Using the Qubit dsDNA HS assay kit (Thermo Scientific, Waltham, USA), the extracted DNA was quantified at $0.2 \mathrm{ng}$ DNA.

\section{Sample and library preparation}

\section{S rRNA amplicon PCR}

The amplicons of the 16S rRNA gene from Methylacidiphilum fumariolicum strain SolV were obtained by PCR of an in-house sample of isolated DNA from M. fumariolicum (Genbank NZ_LM997411) using primers 616F (5'- AGA GTT TGA TYM TGG CTC -'3) and 630R (5'CAKAAAGGAGGTGATCC-'3) with the following settings: $5 \mathrm{~min}$ at $96^{\circ} \mathrm{C}$, followed by 35 cycles of $40 \mathrm{~s}$ at $96^{\circ} \mathrm{C}, 40 \mathrm{~s}$ at $49^{\circ} \mathrm{C}$ and $1 \mathrm{~min}$ at $72^{\circ} \mathrm{C}$ and finalised with an elongation step of 5 $\min$ at $72^{\circ} \mathrm{C}$. The final concentration was measured using the Nanodrop ND-1000 (Isogen, De Meern, The Netherlands).

To reduce the risk of sequencing aspecific PCR-products from the PCR-reaction on genomic DNA, amplicons of the 16S rRNA gene of "Candidatus Kuenenia stuttgartiensis" (GenBank CT573071) were obtained by PCR of an in-house 16S rRNA gene clone using primers pla46 (5'GGATTAGGCATGCAAGTC-'3) and 630R (5'-CAKAAAGGAGGTGATCC-'3) with the following settings: $5 \mathrm{~min}$ at $94^{\circ} \mathrm{C}$, followed by 35 cycles of $40 \mathrm{~s}$ at $96^{\circ} \mathrm{C}, 40 \mathrm{~s}$ at $49^{\circ} \mathrm{C}$ and $1 \mathrm{~min}$ at $72^{\circ} \mathrm{C}$ and finalised with an elongation step of $5 \mathrm{~min}$ at $72^{\circ} \mathrm{C}$. After amplification, the sample was purified from non $16 \mathrm{~S}$ ribosomal DNA by excision and re-extraction of the DNA from a 
$1240.9 \%$ gel (v/w) using the GeneJET gel extraction kit (Thermo Scientific, Waltham, USA)

125 according to manufacturer's protocol. The final concentration was measured using the Qubit

126 dsDNA HS assay kit (Thermo Scientific, Waltham, MA, USA).

\section{Enterobacteria Phage P1}

128 To sequence the P1 phage, 5.1 ng DNA from P1 phage (NC_005856.1) was added to 130 ng of

129 amplified 16S rRNA gene DNA from $M$. fumariolicum strain SolV PCR (referred as P1-spiked

130 sample) and sheared using the Bioruptor ${ }^{\circledR}$ Standard (Diagenode Liege, Belgium) for 10 cycles (1

$131 \mathrm{~min}$ on, $1 \mathrm{~min}$ off) and prepared according to manufacturer's protocol (IonXpress Plus gDNA

132 fragment library preparation Rev C.0, Life).

\section{Infected bioreactor: general protocol}

134 Approximately $7 \mathrm{ng}$ of viral metagenome DNA was spiked with $~ 50 \mathrm{ng}$ of amplified 16S rRNA

135 gene DNA from “ $\mathrm{Ca}$. K. stuttgartiensis" (referred as DNA-spiked-GP sample) and sheared using

136 the Bioruptor for 6 cycles (1 min on, 1 min off) and prepared according to manufacturer's

137 protocol (IonXpress Plus gDNA fragment library preparation Rev C.0, Life).

138

139

140

141

142

143

144

145

146

147

148

149

150

151

152

153

154

155

156

157

158

159

160

\section{Infected bioreactor: specialised protocol}

Approximately $0.1 \mathrm{ng}$ of viral metagenome DNA was spiked with $\sim 43.5 \mathrm{ng}$ of amplified $16 \mathrm{~S}$ rRNA gene DNA from “Ca. K. stuttgartiensis” (referred as DNA-spiked-SP sample) and sheared using the Bioruptor for 6 cycles (1 min on, 1 min off) and prepared according to manufacturer's protocol (IonXpress Plus gDNA fragment library preparation Rev C.0, Life).

\section{Negative control of amplified 16S ribosomal DNA}

Approximately $43.5 \mathrm{ng}$ of amplified 16S rRNA gene DNA from "Ca. K. stuttgartiensis" (referred as neg-16S sample), was sheared using the Bioruptor for 6 cycles (1 min on, 1 min off) and prepared according to manufacturer's protocol (IonXpress Plus gDNA fragment library preparation Rev C.0, Life).

\section{MDA amplification}

Approximately $0.1 \mathrm{ng}$ of DNA from the specialised isolation protocol (i.e. DNA-Spiked-SP) was amplified using the Illustra GenomePhi HY DNA amplification kit (GE Healthcare, Piscataway, NJ, USA) as per manufacturer's protocol. The first amplification round yielded $15 \mathrm{ng}$ of DNA (referred as $1 \mathrm{x}$ MDA sample), Afterwards $10 \mathrm{ng}$ of DNA was used for a second amplification round. This resulted in a yield of $5.4 \mu \mathrm{g}$ DNA (referred as $2 \mathrm{x}$ MDA sample).

For the P1 Phage, 10 ng of DNA was used for amplification (referred as P1-MDA). A negative control was performed using DEPC water instead of DNA (referred to as neg-MDA), resulting in $6.6 \mathrm{ng}$ of DNA. All DNA was cleaned afterwards using the GeneJET plasmid Miniprep kit (Fermentas, Amherst, USA) according to manufacturer's protocol, except for step one, in which $200 \mu 1$ of DEPC was used instead of lysis-buffer.

Starting quantities of DNA and shearing times used for sequence library preparation are given in Table 1. After shearing, the samples were cleaned using a 1:1 volume ratio with AMPure XP 
161 beads (Beckman Coultier, High Wycombe, UK) and further prepared for sequencing as per

162 manufacturer's protocol (IonXpress Plus gDNA fragment library preparation Rev C.0, Life,

163 Carlsbad, USA).

164 Sequencing

165 All samples were sequenced using the Personal Genome Machine Ion Torrent (Thermo

166 Scientific, Waltham, MA, USA) as per manufacturer's protocol. 1 x MDA was sequenced twice, 167 once on a $314 \mathrm{v} 2$ chip and once on a 318v2 chip. All other samples were run on a $318 \mathrm{v} 2 \mathrm{chip}$. All

168 samples were prepared using the Ion PGM ${ }^{\mathrm{TM}}$ Sequencing $400 \mathrm{Kit}$ and Ion PGM ${ }^{\mathrm{TM}}$ Template 169 OT2 400 kit and sequenced with 850 flow cycles.

\section{Bioinformatics}

171 Trimming and mapping to the SILVA database

172 After sequencing all samples were trimmed with quality setting of 0.05 and mismatch of 2 using 173 CLC genomics workbench v. 8 (CLCbio, Aarhus, Denmark). Size trimming was dependent on 174 the dataset and values are given in Table 1. To determine the amount of spiked DNA, the reads 175 from each sample were mapped against the SILVA database 16S rRNA v128 (Yarza et al., 2008) 176 (length 0.5, similarity 0.9).

177 Mapping of P1

178 Trimmed reads from P1-spiked and P1-MDA were mapped against the genome of P1 phage 179 (NC_005856.1) (length 0.5, similarity 0.95) using CLC genomics workbench v. 8 (CLCbio, 180 Aarhus, Denmark).

\section{Case study of infected bioreactor}

182 To remove genomic DNA from the most abundant microorganism in the bioreactor, the trimmed reads from the DNA-spiked sample were mapped against the genome of "Candidatus

184

185

186

187

188

189

190

191

192

193

194

195
Methylomirabilis oxyfera" (Ettwig et al., 2010) (length 0.5, similarity 0.85 ) and the unmapped reads were assembled (word size 22, bubble size 276, contig length 400), using CLC genomics workbench v. 8 (CLCbio, Aarhus, Denmark) (Fig. 1).

The contigs obtained were subsequently mapped against the SILVA database 16S rRNA v119 (length 0.5 , similarity 0.7 ) and contigs that mapped to " $\mathrm{Ca}$. K. stuttgartiensis" were removed from the database, resulting in 4088 remaining contigs. These contigs were checked with ESOM (Ultsch \& Moerchen, 2005) (default settings) and from this, seven clustering contigs with a high depth were obtained and reassembled with SPAdes (v.3.5.0) (Bankevich et al., 2012) using the 'trusted-contigs' and 'careful' settings for those seven contigs.

The reads that were used in this SPAdes re-assembly, were obtained by mapping the complete trimmed spiked DNA dataset to the SILVA database 16S rRNA v119 (length 0.5, similarity 0.7). The reads that did not map were used. 
196 Reassembly with SPAdes created 2094 contigs, 14 of which were bigger than $5000 \mathrm{bp}$. From this 197 set of contigs five putative viral genomes of over $15 \mathrm{kpb}$ could be extracted (197 kbp, $86 \mathrm{kbp}, 71$ $198 \mathrm{kbp}, 41 \mathrm{kbp}$ and $17 \mathrm{kbp}$ ).

199 Assembly of the combined 1 x MDA and 2 x MDA datasets (word size 21, bubble size, 265

200 contig length 1.500 ) resulted in 689 contigs, ranging from 130,897 to 1,504 nucleotides. With the 201 use of ESOM, one putative viral genome over 15kb was identified (42 kbp).

\section{GC content}

203 The plots of the GC-content were created with CLC genomics workbench v. 8.

\section{Peak mixture identification}

205

206

207

208

209

210

211

212

213

214

215

216

217

218

219

220

221

222

223

224

225

226

227

228

229

230

Statistical analysis program R (package mixdist; Macdonald P., 2012) was used to identify the different peaks within DNA-spiked-SP, 1 x MDA and 2 x MDA (Supplement 1).

\section{Differential coverage}

For differential coverage, reads from the DNA-spiked sample were mapped against the SILVA database $16 \mathrm{~S}$ rRNA v119 (length 0.5 , similarity 0.7 ). Unmapped reads were combined with the reads from the second $1 \mathrm{x}$ MDA run and assembled (word 35, bubble size 271, contig length 1.000) with CLC genomics workbench v. 8. Subsequent mapping of each read set (length 0.5 , similarity 0.8 ) was performed against the assembled contigs. The depth of both sets was plotted against one another.

\section{Horizontal coverage}

To assess how much of each virus was present in each set, the trimmed reads from the three sets were mapped against the six putative viral genomes (length 0.5 , similarity 0.95 ). The number of mapped reads and total length was normalised to the size of the dataset and the length of virus, respectively.

\section{Taxonomic analysis}

For an indication of the taxonomic change in the samples, trimmed reads from DNA-spiked-SP, $1 \times$ MDA and $2 \times$ MDA were mapped to the SILVA database 16S rRNA v128. To reduce reads that map solely to the conserved regions of the $16 \mathrm{~S}$ gene, reads shorter than $75 \mathrm{bp}$ for each set were removed before mapping. Reads from DNA-spiked-SP were mapped strictly to reduce false positives (length 0.6 , similarity 0.99 ). Reads that mapped equally well to multiple references were ignored as well as the reads that matched to Planctomycetes, Verrucomicrobia or Chlamydia. Verrucomicrobia and Chlamydia are in the same PVC super-phylum with Planctomycetes and are likely false positives.

Reads from $1 \times$ MDA and 2 x MDA were trimmed less stringent (length 0.5, similarity 0.95), since these samples are not spiked with 16 S ribosomal DNA. Reads that mapped equally well to multiple references were also ignored. 
231 Results

232 DNA from 3 different origins was sequenced after being spiked with DNA from two different

233

234

235

236

237

238

239

240

241

242

243

244

245

246

247

248

249

250

251

252

253

254

255

256

257

258

259

260

261

262

263

264

265

266

267

268

16S rRNA gene sources. The number of reads before and after trimming is outlined in Table 1, as well as the number of reads after mapping to the Silva $16 \mathrm{~S}$ rRNA database.

For the DNA-spiked-GP sample the number of reads was lower than expected (an observed percentage of $1.72 \%$ to an expected percentage of $14.0 \%$ ). The number of reads for the $\mathrm{P} 1-$ spiked sample was slightly higher than expected (5.08\% observed to $3.85 \%$ expected). The number of reads for the DNA-spiked-SP sample was 55 times higher than expected $(12.66 \%$ observed to $0.23 \%$ expected).

\section{Mapping and assembly of P1}

Reads from P1-spiked and P1-MDA samples were both mapped to the genome of P1 phage (NC_005856.1) and the results are overall the same regarding horizontal coverage of the genome. A drop in coverage from basepair 5710 to 15006 for both samples is clearly visible, although in both samples reads do still map to the genome (Fig. 2).

Case study of an infected bioreactorViral DNA extracted from biomass from an infected bioreactor was sequenced following two different approaches: MDA amplification (two samples) and nonamplified DNA spiked with bacterial 16S rRNA gene DNA. This resulted in three datasets comprising of a total of 770,366 trimmed reads for $1 \mathrm{x}$ MDA and 187,178 trimmed reads for $2 \mathrm{x}$ MDA. After the reads from the non-amplified spiked DNA were trimmed and mapped against the SILVA database, the final number of reads left was 529,481. After trimming, GC graphs were created showing the GC distribution of each dataset (Fig. 3) and underlying peaks were identified using the mixdist package from R (Supplement 1). In the DNA-spiked-SP sample a total four peaks were identified $(62 \%, 57 \%, 43 \%$ and $42 \% \mathrm{GC})$, with peaks at $62 \% \mathrm{GC}$ and $57 \%$ $\mathrm{GC}$ as the two most prominent ones (respectively 0.37 and 0.47 out of 1 ). After one round of amplification, this has shifted to $57 \%$ GC (0.67) and $45 \%$ GC (0.24). The peak at $62 \%$ completely disappeared. After two rounds of amplification, the peak at $57 \% \mathrm{GC}$ has been further reduced to 0.08 , while both peaks around 43\% GC (0.69) and 42\% GC (0.23) have increased.

A total of six different viral sequences (putative genomes) could be assembled using the reads from datasets 1 x MDA, 2 x MDA and DNA-spiked-SP by a combination of various methods (see Materials \& methods and Gambelli et al., 2016 for a more elaborate description of the viruses). The DNA-spiking method resulted in five complete viral genomes over $15 \mathrm{kbp}$ in size while from the MDA set only one complete viral genome could be recovered after assembly. The length of the 5 viral genomes extracted from the spiked dataset ranged from $197 \mathrm{kbp}$ to $17 \mathrm{kbp}$ with GC contents from $67 \%$ to $54 \%$. The length of the viral genome extracted from the MDA dataset is $42 \mathrm{kbp}$ with a GC content of 35\%. Five of the viral genomes (197 kbp, $86 \mathrm{kbp}, 71 \mathrm{kbp}$, $42 \mathrm{kbp}$ and $17 \mathrm{kbp}$ ) contained the same sequence on each end of the contig indicating a full circular genome. Fig. 4 shows the comparison of the total amount of virus genomes that could be retrieved from the individual datasets. Fig. 4a shows that all six virus genomes could be 
269

270

271

272

273

274

275

276

277

278

279

280

281

282

283

284

285

286

287

288

289

290

291

292

293

294

295

296

297

298

299

300

301

302

303

304

305

recovered in nearly complete length from the DNA-spiked dataset. In contrast, MDA amplification clearly lowered these percentages. Fig. $4 \mathrm{~b}$ shows the percentage of reads from each viral metagenome mapping to the six virus genomes. With the MDA amplified samples, the percentages of reads mapping is low with a comparable relative abundance. The non-amplified sample not only shows more variation in relative abundance, but the total amount of mapped reads comprises over half of the original dataset. Whit the MDA method, the number of mapped reads drops to lower than 3\%. Fig. 4c shows the average depth of each viral genome after mapping the reads of the individual datasets. The data are comparable to Fig. $4 \mathrm{~b}$. Using the DNA-spiked dataset, high depth $(>35)$ is found for five out of six viral genomes. The MDA datasets show very low depth and only gave better results for the $42 \mathrm{kbp}$ virus.

Assembly of the reads from the DNA-spiked sample and the $1 \mathrm{x}$ MDA sample resulted in a total of 1644 contigs. Differential coverage of these contigs is shown in Fig. 5. From the figure it is clear that the depth for each set of contigs differs completely since two similar sets would result in contigs placed on a diagonal straight line. However, the figure shows an almost perpendicular distribution. Whereas the contigs originating from the DNA-spiked sample are placed in a low horizontal line, the contigs originating from the 1 x MDA sample are vertically placed on the coverage graph. When looking at the location of contigs, it becomes clear that high $\mathrm{GC} \%$ contigs (green) are present within the DNA-spiked dataset but are low in the amplified sample while low GC\% contigs (pink) are much more abundant in the $1 \mathrm{x}$ MDA dataset. The figure also demonstrates the wide range in sequencing depth when no amplification was applied (maximum around 380) in contrast to amplification, as the sample depth was lowered tenfold, with a maximum around 38 .

For the taxonomy, mapping of the reads to the Silva database resulted in 322 reads for DNAspiked-SP. Groups represented by $4 \%$ of the reads or more are Actinobacteria $(22.7 \%)$, Rhizobiales (18\%), Clostridia (7.8\%), Omnitrophica (5.6\%), Nitrospina (5.3\%), Chloroflexi (4.7\%) and Nitrospira (4.0\%) (Table 2). The remaining reads are $18.3 \%$ of the total. For $1 \mathrm{x}$ MDA 92 reads were mapped against WS6 (57.6\%), Microgenomata (18.5\%), SAR11 (5.4\%), Bacillales (5.4\%) and Saccharibacteria (4.7\%). For 2 x MDA 33 reads were mapped against WS6 (45.5\%), Microgenomata (18.2\%), Saccharibacteria (12.1\%) and Woesearchaeota_DHVEG-6 (12.1\%).

\section{Discussion}

Here we describe a case study in which we sequenced low amount of DNA (viral DNA) after spiking 16S rRNA gene DNA and compare this to sequencing after traditional MDA amplification.

For both metagenomes containing viral genomes up to $200 \mathrm{kbp}$ and the single P1 genome of 95 $\mathrm{kbp}$ it was possible to retrieve the original data after spiking with 16S rRNA gene DNA. . For the DNA-spiked-GP, no MDA data were available for an in-depth comparison. However after 
306

307

308

309

310

311

312

313

314

315

316

317

318

319

320

321

322

323

324

325

326

327

328

329

330

331

332

333

334

335

336

337

338

339

340

341

342

mapping reads to the genomes, it was clear that viruses 1 to 6 were present. The distribution was different from the DNA-spiked-SP sample, which can either be caused by bias introduced by the extraction protocol or a change in the viral population.

Comparing P1-spiked and P1-MDA by mapping back their reads to the P1 genome resulted in different depth values, being 4438 for P1-MDA and 65 for P1-spiked, respectively. The horizontal coverage for both sets was the same. This is not unexpected, since there is no preferential genome for the MDA enzyme to amplify. Curiously, the P1 phage used during this experiment seems to have evolved during the time in our lab, as in both the MDA and spiking method a gap appeared at the same locus. However, the depth did not drop to 0 in both samples, indicating that at least a small portion of the original viral population was still present.

Two controls were sequenced along with the samples (Table 1). One control was the 16S rRNA gene DNA without sample DNA. Although no other reads were expected, $0.55 \%$ of the reads was not of $16 \mathrm{~S}$ origin. Since most of those reads matched to M. fumariolicum strain SolV in a BLAST search, the most likely explanation is contamination during library preparation. The level of contamination is not high enough to account for the amount of non-16S in the spiked samples. On top of that, the composition of the non-16S reads is different for the negative control and samples.

The second control consists of a MDA run performed on DEPC-water. In absence of template DNA, MDA still produces dsDNA, since at some point during amplification, the random hexamers act like a template instead of primer. Moreover, MDA is by design very sensitive to small amounts of DNA, therefore any contamination is easily picked up. The contamination however did not consist of the sampled viral or bacterial community.

Differential binning of spiked DNA and the 1 x MDA not only shows a remarkable difference between both samples in terms of composition, but also demonstrates the loss of information about the abundance of contigs in the samples. Depth (vertical coverage) of a contig is a measurement of abundance of that contig in a sample. In the DNA-spiked sample, the maximum depth is 380. In the MDA amplified sample the maximum depth has dropped to 38. Moreover, the best covered contigs after MDA amplification are not the contigs best covered in the spiked DNA. This shift means that sequencing depth after MDA treatment is a poor indicator of abundance of contigs in the original sample. This also complicates binning afterwards, as depth or abundance is often used as a parameter in the binning of metagenomes.

Like in previous reports (Kim \& Bae, 2011; Marine et al., 2014), our experiments show the bias that MDA amplification of metavirome or metagenome DNA introduces into the dataset. In the extreme case of applying MDA twice, the dataset is completely changed with major consequences for results obtained, which as such may not reflect the sampled ecosystem. Although taxonomic analysis is troublesome on a sample that is spiked with short $16 \mathrm{~S}$ ribosomal DNA reads because of the conserved regions, a taxonomic change was observed when analysing 
343 the residual $16 \mathrm{~S}$ genes that were present as contamination in the extracted viral DNA (Table 2).

344 The DNA-spiked-SP showed a variety of organisms present with a few groups in high numbers

345 like Rhizobiales and Actinobacteria. The $1 \times$ MDA and 2 x MDA show a shift towards the WS6

346 group and the group of Microgenomata. Analysis on a sample that is spiked with 16S rRNA gene

347 DNA is not straightforward because of the risk of false positives that are likely to occur.

348 Nonetheless, the change (from $18.9 \%$ to $1.1 \%$ for Rhizobiales and $22.7 \%$ to $0 \%$ for Nitrospira)

349 was so pronounced that false positives are very unlikely the only cause of the shift. Moreover the

350 shift towards $1 \times$ MDA (from $0.6 \%$ to $18 \%$ and the group Microgenomata and from $0 \%$ to $57.6 \%$

351 for the WS6) would be smaller with false positives.

352 Several different reasons have been given in literature regarding the bias of MDA such as

353 fragment length, GC-content, quality of DNA and even different MDA kits (Abulencia et al.,

354 2006; Kim \& Bae, 2011; Yan et al., 2004; Yilmaz et al., 2010). Although there seems to be no

355 general consensus, for our results it seems that specifically DNA with a low GC content is

356 favoured. This may lead to a considerable shift of overall GC content causing a severe

357 underestimation of the quantity of viruses with a higher GC percentage and they might thus be

358 easily overlooked in metagenomic research.

359 From the spiked datasets, it is clear that the final ratio of the sample versus 16S rRNA gene DNA

360 can vary considerably. Possible explanations could be differences in DNA concentration

361 measurements or adjustments made for an optimal sequencing run prior to the final shearing of

362 the sample (Supplement 2). The amount of reported 16S rRNA gene reads can also be influenced

363 by the mapping settings (stringent vs not stringent or local vs global). In practical terms, it is

364 easier to assemble the complete dataset and filter out 16S rRNA gene sequences afterwards. The

$36516 \mathrm{~S}$ ribosomal fragments would be longer and fewer in numbers after assembly and thus easier

366 to identify. However, pre-filtering most of the 16S rRNA gene reads will speed up assembly.

367 A positive side effect of the spiking protocol is that it lowers the risk of contamination. By

368 choosing the 16S rRNA gene of a microorganism which is very uncommon to the laboratory,

369 contamination can easily be recognized and filtered out. One could even use the eukaryotic $18 \mathrm{~S}$

370 rRNA gene in a prokaryotic-based lab and vice versa.

371 In this case study we described a protocol for viral metagenome sequencing of extremely low

372 amounts of DNA(as low as $0.1 \mathrm{ng}$ ) that is unbiased, inexpensive, easy and readily available for

373 any lab with sequencing facilities and that can possibly be extended for other non-16S/18S

374 containing DNA like plasmids, or other Next Generation Sequencing platforms like MinIon or

375 Illumina.

\section{Conclusions}

377 When dealing with low quantities of DNA for Next Generation Sequencing from environmental 378 samples like water or forensic samples, multiple displacement amplification (MDA) of the DNA 
379 from the sample might not be the method of choice to obtain enough starting material. In a case 380 study we have shown that spiking with 16S rRNA gene DNA could be an alternative, eliminating 381 the need for amplification. The reads resembling the added DNA can be easily discarded 382 afterwards and we observed a very low bias in the dataset compared to the MDA method.

\section{Acknowledgements}

384 We thank Simon Guerrero for supplying the bioreactor material.

385

386

387

388

389

390

391

392

393

394

395

396

397

398

399

400

401

402

403

404

405

406

407

408

409

410

\section{References}

Abulencia CB, Wyborski DL, Garcia JA, Podar M, Chen W, Chang SH, Chang HW, Watson D, Brodie EL, Hazen TC, and Keller M. 2006. Environmental whole-genome amplification to access microbial populations in contaminated sediments. Applied Environmental Microbiology 72:3291-3301. 10.1128/AEM.72.5.3291-3301.2006

Bankevich A, Nurk S, Antipov D, Gurevich AA, Dvorkin M, Kulikov AS, Lesin VM, Nikolenko SI, Pham S, Prjibelski AD, Pyshkin AV, Sirotkin AV, Vyahhi N, Tesler G, Alekseyev MA, and Pevzner PA. 2012. SPAdes: A new genome assembly algorithm and its applications to singlecell sequencing. Journal of Computational Biology 19:455-477. 10.1089/cmb.2012.0021

Breitbart M, Salamon P, Andresen B, Mahaffy JM, Segall AM, Mead D, Azam F, and Rohwer F. 2002. Genomic analysis of uncultured marine viral communities. Proceedings of the National Academy of Sciences of the United States of America 99:14250-14255. 10.1073/pnas.202488399

Brum JR, and Sullivan MB. 2015. Rising to the challenge: accelerated pace of discovery transforms marine virology. Nature Reviews Microbiology 13:147-159. 10.1038/nrmicro3404

Clokie MR, Millard AD, Letarov AV, and Heaphy S. 2011. Phages in nature. Bacteriophage 1:31-45. 10.4161/bact.1.1.14942

Cunningham BR, Brum JR, Schwenck SM, Sullivan MB, and John SG. 2015. An inexpensive, accurate, and precise wet-mount method for enumerating aquatic viruses. Applied and Environmental Microbiology 81:2995-3000. 10.1128/Aem.03642-14

Duhaime MB, Deng L, Poulos BT, and Sullivan MB. 2012. Towards quantitative metagenomics of wild viruses and other ultra-low concentration DNA samples: a rigorous assessment and optimization of the linker amplification method. Environmental Microbiology 14:2526-2537. 10.1111/j.1462-2920.2012.02791.x

Ettwig KF, Butler MK, Le Paslier D, Pelletier E, Mangenot S, Kuypers MM, Schreiber F, Dutilh BE, Zedelius J, de Beer D, Gloerich J, Wessels HJ, van Alen T, Luesken F, Wu ML, van de Pas- 
411 Schoonen KT, Op den Camp HJM, Janssen-Megens EM, Francoijs KJ, Stunnenberg H,

412 Weissenbach J, Jetten MSM, and Strous M. 2010. Nitrite-driven anaerobic methane oxidation by

413 oxygenic bacteria. Nature 464:543-548. 10.1038/nature08883

414 Fuhrman JA. 1999. Marine viruses and their biogeochemical and ecological effects. Nature

415 399:541-548. Doi 10.1038/21119

416 Gambelli L, Cremers G, Mesman R, Guerrero S, Dutilh BE, Jetten MSM, Op den Camp HJM, 417 and van Niftrik L. 2016. Ultrastructure and viral metagenome of bacteriophages from an 418 anaerobic methane oxidizing Methylomirabilis bioreactor enrichment culture. Frontiers in 419 Microbiology 7:1740. 10.3389/fmicb.2016.01740

420 Guo P, El-Gohary Y, Prasadan K, Shiota C, Xiao XW, Wiersch J, Paredes J, Tulachan S, and 421 Gittes GK. 2012. Rapid and simplified purification of recombinant adeno-associated virus.

422 Journal of Virological Methods 183:139-146. 10.1016/j.jviromet.2012.04.004

$423 \mathrm{Kim}$ KH, and Bae JW. 2011. Amplification methods bias metagenomic Libraries of uncultured 424 single-stranded and double-stranded DNA viruses. Applied and Environmental Microbiology 425 77:7663-7668. 10.1128/Aem.00289-11

426 Krishnamurthy SR, and Wang D. 2017. Origins and challenges of viral dark matter. Virus 427 Research 239:136-142. 10.1016/j.virusres.2017.02.002

428 Macdonald P. 2012. mixdist: Finite Mixture Distribution Models. R package version 0.5-4

429 Marine R, McCarren C, Vorrasane V, Nasko D, Crowgey E, Polson SW, and Wommack KE. 430 2014. Caught in the middle with multiple displacement amplification: the myth of pooling for 431 avoiding multiple displacement amplification bias in a metagenome. Microbiome 2. Artn $432 \quad 310.1186 / 2049-2618-2-3$

433 R Development Core Team. 2008. R: A language and environment for statistical computing. R 434 Foundation for Statistical Computing, Vienna, Austria. ISBN 3-900051-07-0, URL

435 http://www.R-project.org.

436 Rinke C, Low S, Woodcroft BJ, Raina JB, Skarshewski A, Le XH, Butler MK, Stocker R, 437 Seymour J, Tyson GW, and Hugenholtz P. 2016. Validation of picogram- and femtogram-input 438 DNA libraries for microscale metagenomics. PeerJ 4:e2486. 10.7717/peerj.2486

439 Rosario K, and Breitbart M. 2011. Exploring the viral world through metagenomics. Current 440 Opinion in Virology 1:289-297. 10.1016/j.coviro.2011.06.004

441 Speth DR, In 't Zandt MH, Guerrero-Cruz S, Dutilh BE, and Jetten MS. 2016. Genome-based 442 microbial ecology of anammox granules in a full-scale wastewater treatment system. Nature 443 Communications 7:11172. 10.1038/ncomms11172 
444 Steward GF, Culley AI, Mueller JA, Wood-Charlson EM, Belcaid M, and Poisson G. 2013. Are 445 we missing half of the viruses in the ocean? ISME Journal 7:672-679. 10.1038/ismej.2012.121

446 Thurber RV, Haynes M, Breitbart M, Wegley L, and Rohwer F. 2009. Laboratory procedures to 447 generate viral metagenomes. Nature Protocols 4:470-483. 10.1038/nprot.2009.10

448 Ultsch A, Moerchen F. 2005. ESOM-Maps: tools for clustering, visualization, and classification 449 with Emergent SOM, Technical Report Dept. of Mathematics and Computer Science, University 450 of Marburg, Germany, No. 46

451 Yan J, Feng J, Hosono S, and Sommer SS. 2004. Assessment of multiple displacement 452 amplification in molecular epidemiology. Biotechniques 37:136-138, 140-133

453 Yarza P, Richter M, Peplies J, Euzeby J, Amann R, Schleifer KH, Ludwig W, Glockner FO, and 454 Rossello-Mora R. 2008. The All-Species Living Tree project: a 16S rRNA-based phylogenetic 455 tree of all sequenced type strains. Systematic and Applied Microbiology. 31:241-250.

456 10.1016/j.syapm.2008.07.001

457 Yilmaz S, Allgaier M, and Hugenholtz P. 2010. Multiple displacement amplification 458 compromises quantitative analysis of metagenomes. Nature Methods 7:943-944.

$45910.1038 /$ nmeth1210-943 
460

461

462

463

464

465

466

467

468

469

470

471

472

473

474

475

476

477

478

479

480

481

482

483

484

485

486

487

488

489

490

491

492

493

\section{Legends to the figures}

Figure 1: Overview of the protocols used to compare MDA and 16S ribosomal DNA spiking. After DNA isolation, half of the sample was amplified using MDA, while the other half was spiked with $16 \mathrm{~S}$ ribosomal DNA. Contigs and reads containing 16S ribosomal DNA were filtered out through mapping to the Silva database.

Figure 2: Coverage of the Enterobacteria P1 phage after sequencing using MDA amplification and $16 \mathrm{~S}$ ribosomal DNA spiking.

Figure 3: Distribution of reads based on their individual GC content (\%) from three different sample preparations. A. DNA-spiking protocol; B. 1 x MDA amplification; C. 2 x MDA amplification. GC graphs were created using CLCgenomics workbench. For statistical evaluation see also Supplement 1.

Figure 4: Comparison of the viral reads from the individual datasets mapping to the six assembled virus genomes (Green, DNA-spiked sample; Blue, 1 x MDA; Red, 2 x MDA). A. Horizontal coverage of the viral genomes with reads from the individual datasets. B. Distribution of the reads from the individual datasets over the assembled viral genomes. C. Depth (vertical coverage) of the viral genomes with reads from the individual datasets as a measure of abundance.

Figure 5: Differential coverage of the viral contigs assembled using a combination of the DNAspiked sample and the $1 \mathrm{x}$ MDA sample with each individual read sets. Each circle represents a contig present after assembly and the placement in the plot shows the abundance of the contig for each read set. Two similar read sets would result in a diagonal straight line. GC content of the different contigs is indicated as depicted in the colour scale and the size of the bubble depicts the length of the contig. Three outliers caused by the MDA amplification method are not shown in the plot.

Table 1: Overview of the general sequencing preparation and results.

Table 2: Table 2: Overview of the 16S reads in DNA-spiked-SP, 1x MDA and 2x MDA in number of reads and percentage. The percentages are colour-coded in a gradient from $0 \%$ (red) to $100 \%$ (green). The reads were identified by mapping to the SILVA 16 S rRNA database v128. DNA-spiked-SP (length 0.6, similarity 0.99): 1x MDA and 2 x MDA (length 0.5, similarity 0.95). Ambiguous reads were removed from the set.

\section{Supplementary Material}

Supplement 1: Statistical evaluation of the peaks shown in Figure 3 using R-package mixdist. From left to right are shown: the graphs, R-code and the final peak statistics. The statistics show 
494 the mean (mu) GC content for a peak, the SD of the peak (sigma) and proportion of the peaks of 495 the total (pi).

496 Supplement 2: Shearing patterns of the initial shearing tests performed to estimate the optimum 497 conditions.

498

499 


\section{Figure 1 (on next page)}

Overview of the methods used to compare MDA and 165 ribosomal DNA spiking.

After DNA isolation, half of the sample was amplified using MDA, while the other half was spiked with 16S ribosomal DNA. Contigs and reads containing 165 ribosomal DNA were filtered out through mapping to the Silva database. 


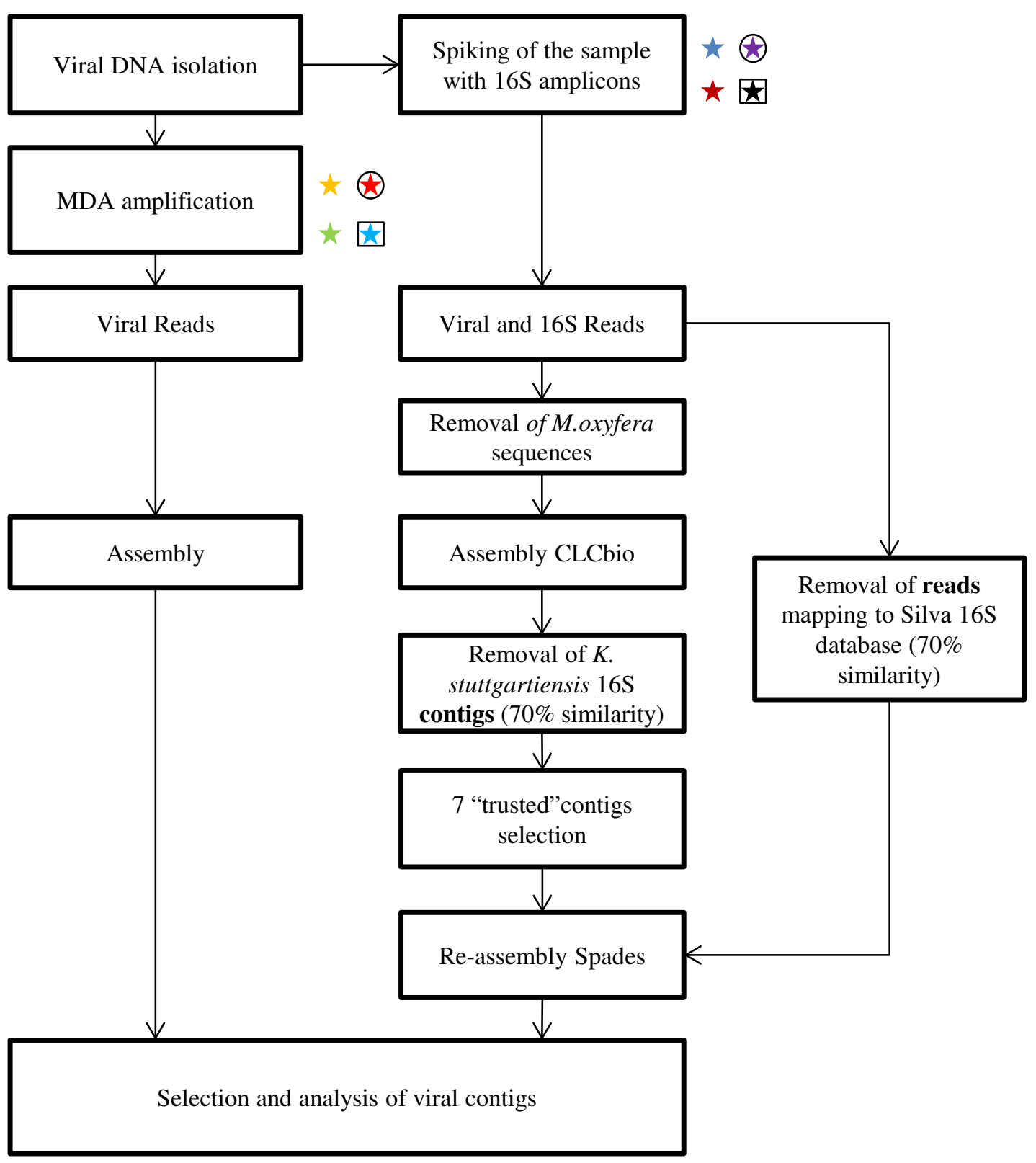

^ DNA-spiked-GP: DNA spiked with 16S ribosomal DNA; General

Protocol

$\star$ DNA-spiked-SP: DNA spiked with 16S ribosomal DNA; Specialised Protocol

$\star$ MDA 1x: 1 x MDA Amplified DNA

$\star$ MDA 2x: 2 x MDA Amplified DNA

图 P1 MDA: P1 faag MDA amplified

因 P1 spiked: P1 faag spiked with 16S ribosomal DNA

๑ Neg 16S: 16S ribosomal DNA

(A) MDA: MDA amplification of DEPC 


\section{Figure 2 (on next page)}

Coverage of the Enterobacteria P1 phage after sequencing using MDA amplification and 16S ribosomal DNA spiking. 


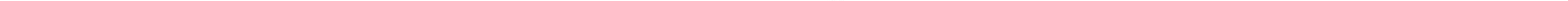




\section{Figure 3 (on next page)}

Distribution of reads obtained from lon Torrent sequencing using three different sample preparation methods based on their individual GC content in \% of the total number of reads in one sample. 


\section{Figure 4 (on next page)}

Comparison of the viral reads from the individual datasets mapping to the six assembled virus genomes (Green, DNA-spiked sample; Blue, 1 × MDA; Red, 2 × MDA).

A. Horizontal coverage of the viral genomes with reads from the individual datasets. B. Distribution of the reads from the individual datasets over the assembled viral genomes. C. Depth (vertical coverage) of the viral genomes with reads from the individual datasets as a measure of abundance. 
GC content
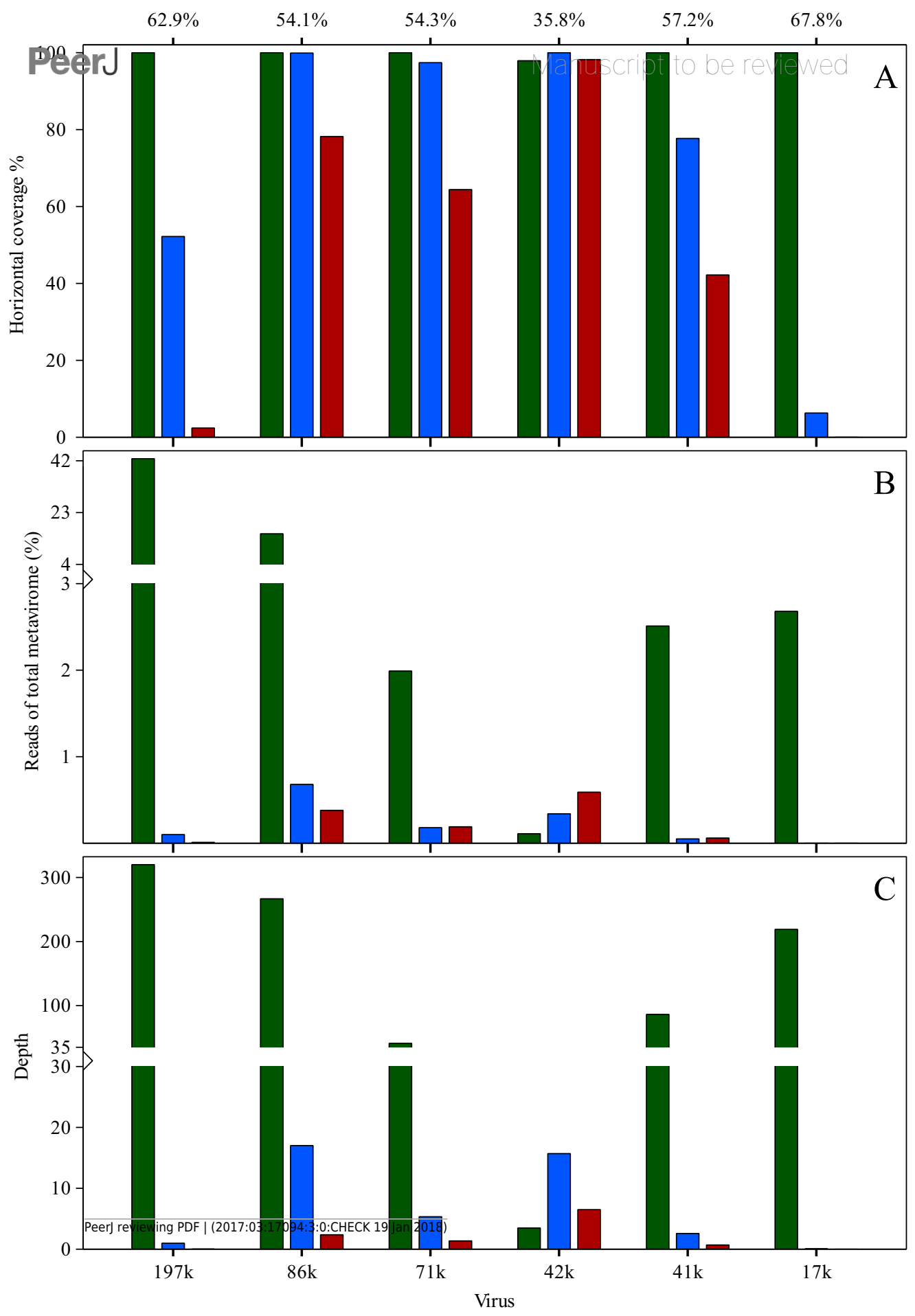


\section{Figure 5 (on next page)}

Differential coverage of the viral contigs assembled using a combination of the DNAspiked sample and the 1 x MDA sample with each individual read sets.

Each circle represents a contig present after assembly and the placement in the plot shows the abundance of the contig for each read set. Two similar read sets would result in a diagonal straight line. GC content of the different contigs is indicated as depicted in the colour scale and the size of the bubble depicts the length of the contig. Three outliers caused by the MDA amplification method are not shown in the plot. 


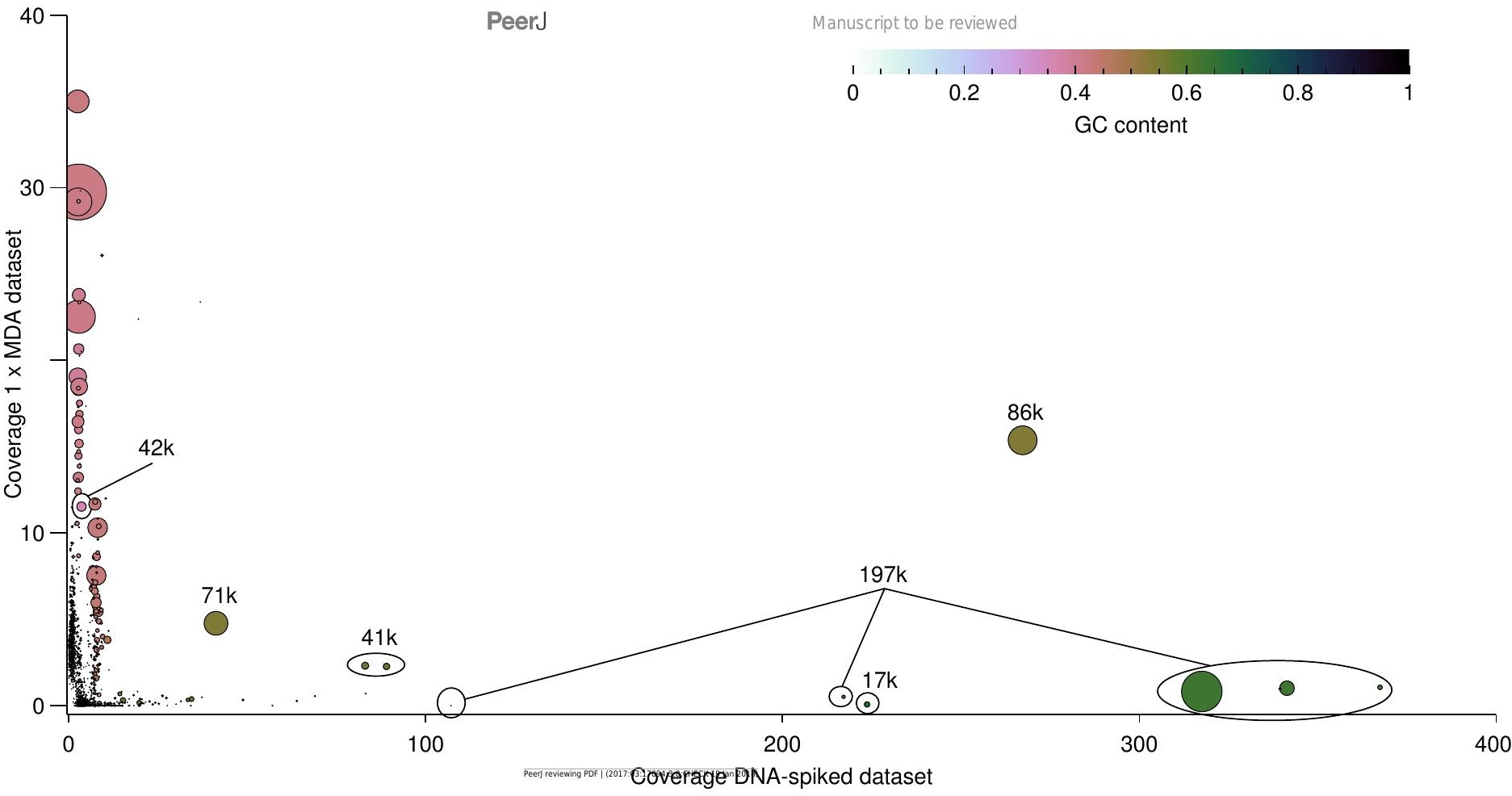




\section{Table $\mathbf{1}$ (on next page)}

Overview of the parameters and results from sequencing, trimming and mapping to the 6 viral sequences and P1 phage.

a obtained from Qubit measurements

${ }^{b}$ quality trimming $=0.05$, ambiguous nucleotide limit $=2$

c mapping settings $=$ local; 0.5 length; 0.9 similarity

d mapping settings= local; 0.5 length; 0.95 similarity

e only remaining reads used

$\mathrm{NA}=$ not applicable 


\begin{tabular}{|c|c|c|c|c|c|c|c|c|c|c|c|c|}
\hline Sample $^{a}$ & $\begin{array}{c}\text { DN } \\
\text { A } \\
(\mathbf{n g})^{b}\end{array}$ & $\begin{array}{c}16 S \\
(\mathrm{ng}) \\
\mathrm{b}\end{array}$ & $\begin{array}{l}\text { Shearing } \\
\text { cycle; } 1 \text { min } \\
\text { on } / 1 \text { min off }\end{array}$ & \# reads & $\begin{array}{l}\text { Trimming } \\
\text { settings }\end{array}$ & $\begin{array}{c}\text { \# } \\
\text { Trimme } \\
\text { d reads }\end{array}$ & $\begin{array}{c}\text { Mapped } \\
\text { reads to } \\
16 S^{d} \\
\end{array}$ & $\underset{\text { g reads }}{\text { Remainin }}$ & $\begin{array}{l}\text { Expected } \\
\text { \% of non } \\
16 \mathrm{~S} \text { reads } \\
\end{array}$ & \begin{tabular}{|c|} 
Observed \\
$\%$ non $16 \mathrm{~S}$ \\
reads \\
\end{tabular} & $\begin{array}{c}\% \\
\text { mapped } \\
\text { to \#1-6 } \\
\end{array}$ & $\begin{array}{c}\% \\
\text { mappe } \\
\text { d to P1 } \\
\end{array}$ \\
\hline neg-16S & 0 & 43.5 & $6 x$ & 58989 & $25-375 b p$ & 58616 & 58293 & 323 & 0.00 & 0.55 & $0.00^{\mathrm{e}}$ & $0,00^{\mathrm{e}}$ \\
\hline P1-spiked & 5 & 130 & $10 x$ & 543649 & $25-400$ & 522819 & 496250 & 26569 & 3.85 & 5.08 & $0.02^{\mathrm{e}}$ & $31.82^{\mathrm{e}}$ \\
\hline P1-MDA & 100 & NA & $6 x$ & 373351 & $\begin{array}{c}<25 \mathrm{bp} ; 25- \\
325 \mathrm{bp}\end{array}$ & 327451 & 36 & 327415 & 100 & 99.99 & 0.00 & 94.83 \\
\hline $\begin{array}{c}\text { DNA-spiked- } \\
\text { GP }\end{array}$ & 7 & 50 & $6 x$ & $\begin{array}{c}476080 \\
7 \\
\end{array}$ & $\begin{array}{c}25-400 \mathrm{bp} \\
25-350 \mathrm{bp}\end{array}$ & 4636949 & 4557290 & 79659 & 14.00 & 1.72 & $31.31^{\mathrm{f}}$ & $0.01^{\mathrm{f}}$ \\
\hline $\begin{array}{c}\text { DNA-spiked- } \\
\text { SP }\end{array}$ & 0.1 & 43.5 & $6 x$ & $\begin{array}{c}433446 \\
0\end{array}$ & & 4268134 & 3727603 & 540531 & 0.23 & 12.66 & $64.43^{\mathrm{f}}$ & $0.53^{f}$ \\
\hline $1 \times \mathrm{MDA}$ & 5.1 & NA & $6 x$ & 797971 & $\begin{array}{c}25-325 \mathrm{bp} ; \\
25-400 \mathrm{bp} \\
\text { and } 15 \mathrm{bp} \text { on } \\
5 \text {, end }\end{array}$ & 770366 & 3811 & 766555 & 〜99.9 & 99.51 & 1.34 & 0.15 \\
\hline $2 \times \mathrm{MDA}$ & 100 & NA & $6 x$ & 190509 & $25-375 b p$ & 187178 & 1667 & 185511 & $\sim 99.9$ & 99.11 & 1.24 & 0.09 \\
\hline neg-MDA & 100 & NA & $9 \mathrm{x}$ & 94905 & $25-340 \mathrm{bp}$ & 93407 & 775 & 92632 & 100 & 99.17 & 0.00 & 0.00 \\
\hline
\end{tabular}

Table 1: Overview of the parameters and results from sequencing, trimming and mapping to the 6 viral sequences and P1 phage.

${ }^{\text {a }}$ P1 refers to phage P1; GP refers to DNA isolated using the general protocol; SP refers to DNA isolated using the specialized protocol

${ }^{b}$ obtained from Qubit measurements

${ }^{\mathbf{c}}$ quality trimming $=0.05$, ambiguous nucleotide limit $=2$

d mapping settings $=$ local; 0.5 length; 0.9 similarity

${ }^{\mathrm{e}}$ mapping settings $=$ local; 0.5 length; 0.95 similarity

f only remaining reads used

$\mathrm{NA}=$ not applicable 


\section{Table 2 (on next page)}

Overview of the 165 reads inDNA-spiked-SP, $1 \times$ MDA and 2x MDA in number of reads and percentage.

The percentages are colour-coded in a gradient from $0 \%$ (red) to $100 \%$ (green). The reads were identified by mapping to the SILVA 16S rRNA database v128. DNA-spiked-SP (length 0.6 , similarity 0.99 ): $1 \times$ MDA and $2 \times$ MDA (length 0.5 , similarity 0.95 ). Ambiguous reads were removed from the set. 
1 Table 2: Overview of the 16S reads in DNA-spiked-SP, 1x MDA and 2x MDA in number of 2 reads and percentage.

\begin{tabular}{|c|c|c|c|c|c|c|}
\hline & \multicolumn{2}{|c|}{ DNA-spiked-SP } & \multicolumn{2}{|c|}{$1 \times$ MDA } & \multicolumn{2}{|c|}{$2 \times$ MDA } \\
\hline & \# & $\%$ & $\#$ & $\%$ & \# & $\%$ \\
\hline Rhizobiales & 61 & 18.9 & 1 & 1.1 & 0 & 0.0 \\
\hline OPB41 & 2 & 0.6 & 0 & 0.0 & 0 & 0.0 \\
\hline Actinomycetales & 3 & 0.9 & 0 & 0.0 & 0 & 0.0 \\
\hline SAR11 & 6 & 1.9 & 5 & 5.4 & 1 & 3.0 \\
\hline Latescibacteria & 7 & 2.2 & 0 & 0.0 & 0 & 0.0 \\
\hline Clostridia & 25 & 7.8 & 0 & 0.0 & 0 & 0.0 \\
\hline Saccharibacteria & 7 & 2.2 & 4 & 4.3 & 4 & 12.1 \\
\hline Omnitrophica & 18 & 5.6 & 0 & 0.0 & 0 & 0.0 \\
\hline Streptomycales & 12 & 3.7 & & 0.0 & 0 & 0.0 \\
\hline Microgenomates & 2 & 0.6 & 17 & 18.5 & 6 & 18.2 \\
\hline WS6 & 0 & 0.0 & 53 & 57.6 & 15 & 45.5 \\
\hline Woesearchaeota DHVEG-6 & 0 & 0.0 & 1 & 1.1 & 4 & 12.1 \\
\hline Parcubacteria & 1 & 0.3 & 1 & 1.1 & 0 & 0.0 \\
\hline Lactobacillales & 0 & 0.0 & 2 & 2.2 & 0 & 0.0 \\
\hline Staphylococcaceae & 1 & 0.3 & 2 & 2.2 & 0 & 0.0 \\
\hline Bacillales & 0 & 0.0 & 5 & 5.4 & 0 & 0.0 \\
\hline Chloroflexi & 15 & 4.7 & 0 & 0.0 & 0 & 0.0 \\
\hline Pseudomonadales & 0 & 0.0 & 0 & 0.0 & 1 & 3.0 \\
\hline Thaumarchaeota & 0 & 0.0 & 0 & 0.0 & 1 & 3.0 \\
\hline Nitrospira & 13 & 4.0 & 0 & 0.0 & 0 & 0.0 \\
\hline Actinobacteria & 73 & 22.7 & 0 & 0.0 & 0 & 0.0 \\
\hline Nitrospina & 17 & 5.3 & 0 & 0.0 & 0 & 0.0 \\
\hline Cyanobacteria & 0 & 0.0 & 1 & 1.1 & 1 & 3.0 \\
\hline Other & 59 & 18.3 & 0 & 0.0 & 0 & 0.0 \\
\hline Total read count & 322 & 100.0 & 92 & 100 & 33 & 100 \\
\hline
\end{tabular}

3 The percentages are colour-coded in a gradient from $0 \%$ (red) to $100 \%$ (green). The reads were 4 identified by mapping to the SILVA 16S rRNA database v128. DNA-spiked-SP (length 0.6, 5 similarity 0.99): $1 \mathrm{x}$ MDA and 2 x MDA (length 0.5, similarity 0.95). Ambiguous reads were 6 removed from the set. 